\title{
机器人多指灵巧手的研究现状、趋势与挑战*
}

蔡世波 ${ }^{1,2}$ 陶志成 ${ }^{1,2}$ 万伟伟 ${ }^{3}$ 喻豪勇 ${ }^{4}$ 鲍官军 ${ }^{1,2}$

(1. 浙江工业大学机械工程学院 杭州 310023;

2. 浙江工业大学特种装备制造与先进加工技术教育部/浙江省重点实验室 杭州 310023;

3. 大阪大学基础工学研究科 大阪 560-8531 日本;

4. 新加坡国立大学生物医学工程系 新加坡 117575 新加坡)

摘要: 机器人多指灵巧手是一种高度灵活、复杂的末端执行器, 因其能够模仿人手的各种灵巧抓持和复杂操作能力, 半个多 世纪以来得到持续的研发投入和广泛关注, 备受社会各界期待。综述分析了仿人型机器人多指灵巧手的演化过程、研究与开 发现状。从仿生结构、驱动、传动、感知、复合/智能材料、建模与控制等方面分析了机器人多指灵巧手的本体复杂性, 在部 分功能复现、灵巧操作功能复现和人手功能的增强三个层次上分析了机器人多指灵巧手的应用复杂性, 进而论述了基于多指 灵巧手的复杂应用简化实现模式, 阐明了机器人多指灵巧手的复杂性与易用性的辩证关系。最后从深度仿生、柔性感知技术、 操作过程规划与控制策略、成本控制四个方面分析了多指灵巧手本体研究的趋势与挑战。

关键词: 多指灵巧手; 仿生; 柔性感知; 操作规划; 建模与控制

中图分类号: TP24

\section{Multi-fingered Dexterous Hands: From Simplicity to Complexity and Simplifying Complex Applications}

\section{CAI Shibo ${ }^{1,2}$ TAO Zhicheng ${ }^{1,2}$ WAN Weiwei $^{3} \quad$ YU Haoyong $^{4}$ BAO Guanjun ${ }^{1,2}$}

(1. College of Mechanical Engineering, Zhejiang University of Technology, Hangzhou 310023;

2. Key Laboratory of Special Purpose Equipment and Advanced Processing Technology, Ministry of Education and Zhejiang Province, Zhejiang University of Technology, Hangzhou 310023;

3. Graduate School of Engineering Science, Osaka University, Osaka 560-8531, Japan;

4. Department of Biomedical Engineering, National University of Singapore, Singapore 117575 Singapore)

\begin{abstract}
A multi-fingered dexterous hand is a highly flexible and complex end-effector for robots. Since it can imitate various dexterous grasping and complex manipulation abilities of human hand, multi-fingered dexterous hand has been continuously studied and developed for more than half a century and has attracted extensive attention and received wide expectation from the public. In this paper, the research and development of humanoid multi-fingered dexterous hands are reviewed and analyzed. The complexity of multi-fingered dexterous hand is analyzed from aspects of bionic structures, actuation systems, transmission, sensing, composite/smart materials, and modeling and control. The complexity of multi-fingered dexterous hand is discussed from an applicational viewpoint in three levels: Partial function repetition, agile manipulation imitation, and function enhancement of human ability. Simplified realization of the complex application based on multi-fingered dexterous hand is also discussed. The dialectical relationship between the complexity of multi-fingered dexterous hand and the simplicity of application is illustrated. Finally, the trend and challenges of the multi-fingered dexterous hand research are analyzed concerning bionics, flexible sensing technology, manipulation planning, control strategy, and reducing cost.
\end{abstract}

* 国家自然科学基金(51775499)、国家重点研发计划(2020YFB130195)和浙 江省属高校基本科研业务费(RF-C2019004)资助项目。20201028 收到初 稿, 20210204 收到修改稿 
Key words: multi-fingered dexterous hands; bionics; flexible sensing; manipulation; modeling and control

\section{0 前言}

作为一种智能化装备, 机器人(特别是仿人型机 器人)在结构、外形、运动、感知、操作等诸多方面 日趋接近人类。随着机器人向工业深度应用和服务、 健康、军事、航空航天等领域的不断拓展, 机器人 与目标对象和环境的交互需求和能力越来越高 ${ }^{[1-2]}$ 。 一般而言, 机器人与环境交互的方式主要包括: 移 动行走、视觉等信息的获取和决策的执行输出。其 中操作和动作决策的执行输出工具在机器人学领域 被称之为末端执行器(End-Effector)。末端执行器是 机器人执行部件的统称, 一般安装于机器人腕部的 末端, 是直接执行任务的装置。末端执行器作为机 器人与环境相互作用的最后环节与执行部件, 对提 高机器人的柔性和易用性有着极为重要的作用, 其 性能的优劣在很大程度上决定了整个机器人的工作 性能。按其功能可以分为两大类, 即: 工具类和抓 手类。

工具类末端执行器是根据具体工作需求专门设 计并预留标准化接口的机器人专用工具, 可以直接 实现具体的加工工种、生产工艺或日常动作, 包括: 喷枪、涂胶枪、点焊机、弧焊焊枪、毛刺打磨机、 铆钉枪、体温枪、手术刀具、吸盘等。

抓手类机器人末端执行器恰如人的双手, 担负 着执行各种动作、抓持和操作的任务。早期的抓手 类末端执行器以气动、液压或电动夹手为主, 应用 于工业流水线进行简单的夹持和搬运工作。为适应 工业现场加工工艺的复杂化和工位集成化，三指、 多自由度的手型末端执行器应运而生。随着机器人 向辅助人工、代替工人、人机合作的工业应用方向 发展, 以及服务机器人、健康辅助机器人、军用机 器人及航天机器人的出现和日益受到关注, 仿人型 的多指灵巧手越来越受到科研单位、创新企业和社 会各界的重视。从近年来的文献分析可知 ${ }^{[3-4]}$, 多指 灵巧手已经成为机器人领域的一大研究热点, 其应 用需求已经凸显并日益增大。机器人多指灵巧手虽 然在自身结构、材料、驱动、传动、感知、建模与 控制等方面日渐精细和复杂, 但预期可以实现的拟 人化强力抓持、精准抓取和灵活操作能力, 将大幅 度降低用户的系统设计与集成、调试控制等繁杂圥 长的工作量, 将目前由系统工程师、研发人员针对 具体应用进行的工艺分析、节拍分解、工位设计等
复杂自动化系统设计与集成, 机器人操作过程编程 与仿真等工作集中由多指灵巧手实现，极大地简化 系统复杂度和设计难度。可见, 多指灵巧手在机器 人系统中是关键部件之一, 并且具有日趋广泛的应 用需求和市场前景。

本文将沿着历史发展的脉络综述分析抓手类机 器人末端执行器的研究与开发工作, 重点总结评述 机器人多指灵巧手的研发现状、应用前景、复杂性 与易用性的辩证关系，探讨多指灵巧手本体研究的 挑战及发展趋势。

\section{1 从夹持器到多指灵巧手}

\section{1 两指夹持器}

夹持器是抓持物体进而操控物体的装置 ${ }^{[5]}$, 模 仿的是手指的夹持运动。它能够在执行某些动作的 同时夹住和松开目标物体。应用于机器人的末端夹 持器一般采用电机或气缸作为驱动, 机构原理以多 连杆机构和气缸为主, 如图 1 所示。其中使用最广 的两指夹持器是气缸式夹持器, 它本质上是一个以 压缩空气为动力的气缸, 如图 $1 \mathrm{~b}$ 所示。当充气时, 夹持器夹指将接近物体并牢牢抓住物体, 以便执行 其他操作, 而当充气方向改变时, 夹持器将松开该 物体。典型应用是改变物体方向或在选放操作中移 动物体。该类夹持器已有多种标准化的产品系列, 例如德国 SCHUNK 公司的气动平行爪夹持器、 FESTO 公司的气动夹持器、亚德客手指气缸、 Gimatic 公司气动手指夹爪、Weiss Robotics 公司气 动夹爪等。

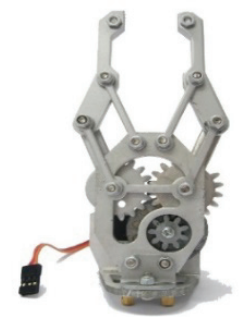

(a) 多连杆夹持器

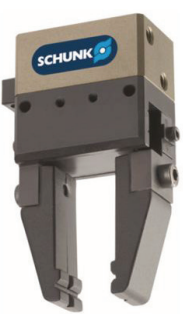

(b) 气缸夹持器
图 1 典型的两指夹持器

两指夹持器结构简单, 运动形式单一稳定, 工 作可靠。在工业现场被大量应用于目标零部件的夹 取、搬运、换位、装配等工位。当然, 其不足之处 也显而易见：缺乏手指的灵活性，不能对复杂形状 的目标进行抓持, 更无法对目标物体实施操作。 


\section{2 多指抓持手}

对于结构复杂或缺少定位基准的目标物体, 两指 夹持器经常会出现拾取失败、甚至无法夹取的现象。 因此出现了三指或者四指抓持手, 主要包括联动型抓 持手、多关节手指抓持手、软体多指抓持手等。

联动型抓持手一般为三指或四指结构 ${ }^{[6]}$, 其基 本原理与前述两指夹持器一样, 由多连杆驱动或气 缸驱动实现多指的同步运动, 如图 2 所示。由于采 用多指设计, 可以对目标物体实施多触点抓持, 提 高了抓持的成功率和可靠性。但是依然缺乏灵活性 和目标物体的适应性。

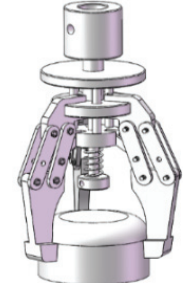

(a) 多连杆三指夹持器

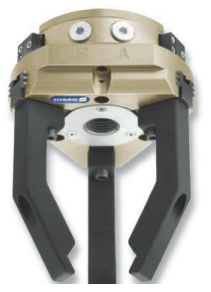

(b) 气缸三指夹持器
图 2 联动型三指夹持器

多关节手指抓持手在其手指上设置多个关节， 虽然多数情况下是被动关节, 但是手指因此而具有 了对目标物体进行仿形接触和抓持的可能性, 扩大 了抓持范围, 并且能够进一步提高抓持的稳定性和 可靠性。因此该原理为众多的机器人手设计所采纳。 该类抓持手的典型代表是 Barrett Technology 公司的 三指 Barrett Hand。如图 3a 所示, Barrett Hand 每个 手指具有 2 个转动自由度, 并且其中 3 个手指的相 对位置可以通过编程控制 ${ }^{[7]}$ 。市场上已出现多款多 关节手指抓持手产品, 例如 Righthand Robotics 公司 的 ReFlex TakkTile 三指手 ${ }^{[8]}$ 、苏州钧舵机器人有限 公司的均巧三指手、德国 SCHUNK 公司的 3-finger gripper hand SDH 等, 分别如图 3b、3c、3d 所示。

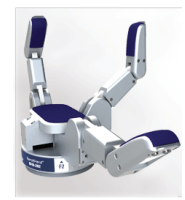

(a) Barrett Hand

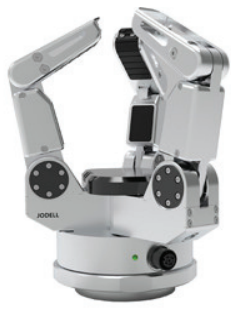

(c) 均巧三指手

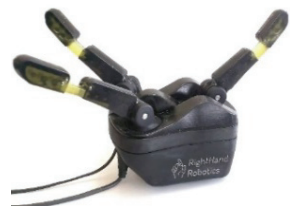

(b) ReFlex TakkTile

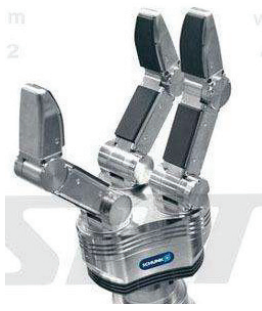

(d) $\mathrm{SDH}$ 手
图 3 多关节手指抓持手
与传统的 “硬” 机器手相比, 软体多指抓持手 在抓取物体时可根据物体形状更自然妥帖地调整形 态。使其在操作易碎品或不规则物体(如鸡蛋、蔬果、 个性化零件等)方面独具优势。早在 1990 年代初, 日本东芝公司利用他们开发的三自由度 FMA 设计 制作了四指柔性手 ${ }^{[9]}$, 如图 4a 所示。浙江工业大学 在国内较早开展软体抓持手的研究与开发工作, 如 球果采摘手 ${ }^{[10]}$ 、黄瓜采摘手 ${ }^{[11]}$ 等农业采摘软体手, 如图 4b、4c 所示。日本立命馆大学则面向食品抓持 配送等需求研发了四指软体手 ${ }^{[12]}$, 如图 $4 \mathrm{~d}$ 所示。 随着软体机器人成为近年来的热点研究领域 ${ }^{[13]}$, 软 体多指抓手得到了更多的关注和研究 ${ }^{[14]}$, 并已形成 产品, 例如美国 Soft Robotics 公司的 mGrip、北京 软体机器人科技有限公司的柔性夹爪、苏州柔触机 器人科技有限公司面向不同行业的软体夹爪组合、 美国 Ubiros Inc 公司的软体抓手等, 如图 5 所示。 图 $5 \mathrm{a} \sim 5 \mathrm{c}$ 所示的软体抓手的手指结构采用外侧气 腔阵列方式实现单向抓持驱动, 抓手的张开动作依 靠硅胶弹性材料的弹性被动实现; 而图 5d 所示的软 体抓手则采用内外双侧气腔阵列方式实现双向主动 驱动, 提高了抓手的动作效率。

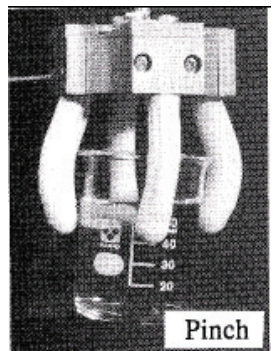

(a) 基于 FMA 的四指手

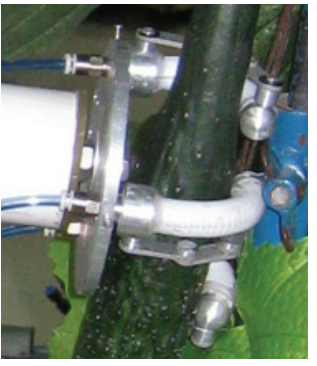

(c) 黄瓜采摘手

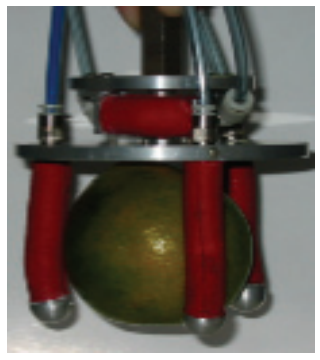

(b) 球果采摘手

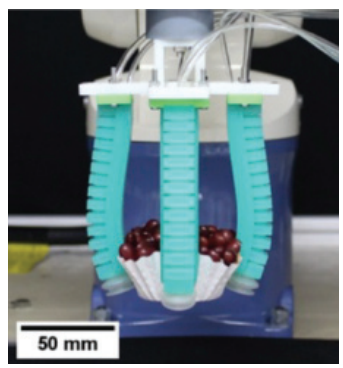

(d) 食品抓持软体手
图 4 软体多指抓持手样机

相比于两指夹持器, 多指抓持手在目标适应性、 抓持稳定性等方面已经有了大幅度的提升, 并且得 到了企业界和用户的认可。然而, 这类多指手的功 能依然停留在实现目标物体的抓持范畴, 还不具有 对目标物体的操作能力。 


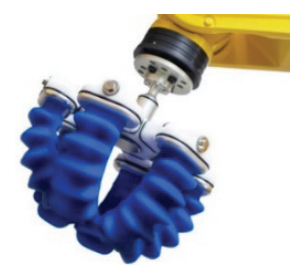

(a) mGrip

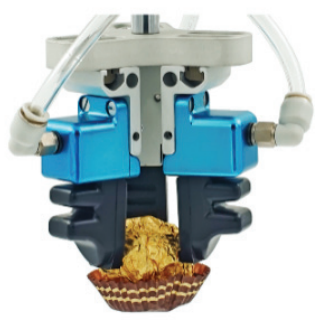

(c) 食品行业夹爪

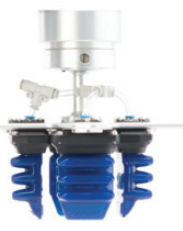

(b) 柔性夹爪

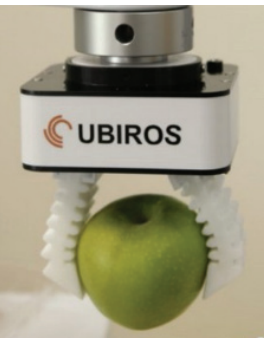

(d) UBIROS gripper
图 5 气动软体多指抓手产品

\section{3 多指灵巧手}

多指灵巧手的机构形式是多指多关节, 并且最 普遍的是手指数目为 $3 \sim 5$ 个, 各手指具有 3 个关节, 手指关节的运动副都是采用转动副。同时, 研究人 员提出了各种协调控制、抓取规划方法用于控制多 指灵巧手实现模拟人手的抓取和操作。可见, 机器 人多指灵巧手以人手的结构和功能为模仿对象, 其 研究的最终目标也是期望能够像人手那样对生产、 生活乃至自然界中的各种物体进行稳定并且灵活的 抓持和操作(纯软体结构的多指手在目前的技术条 件下尚不能实现灵巧运动控制, 不在本文讨论范围 之内)。

机器人多指灵巧手的研究始于 20 世纪 70 年代。 日本 “电子技术实验室” 的 Okada 灵巧手 ${ }^{[15]}$ (图 6a) 具有 3 个手指和一个手掌, 拇指有 3 个自由度, 另 外两个手指各有 4 个自由度, 采用电机驱动和肌腱 传动方式。 20 世纪 80 年代初美国斯坦福大学研制 成功了 Stanford/JPL 灵巧手 ${ }^{[16]}$ ，如图 $6 \mathrm{~b}$ 所示, 该 手有 3 个手指, 每指各有 3 自由度, 采用 12 个直流 伺服电机作为关节驱动器, 采用 $\mathrm{N}+1$ 型腱驱动系统 传递运动和动力。与 Okada 手相比, Stanford/JPL 手的灵活性有较大的改善, 但其控制系统也更为复 杂。美国麻省理工学院和犹他大学联合研制了 $\mathrm{Utah} / \mathrm{MIT}$ 灵巧手 ${ }^{[17]}$, 如图 6c 所示, 具有完全相同 的 4 个手指, 每个手指有 4 个自由度, 采用 $2 \mathrm{~N}$ 型 腱驱动系统传递运动和动力, 整手有 16 个关节, 驱 动器数量达到了 32 个。上述三只灵巧手是该领域研 究初始阶段的典型代表, 为后续仿人型多指灵巧手 研究建立了理论基础

20 世纪末, 随着嵌入式硬件的发展, 多指灵巧
手的研究向着高系统集成度和丰富的感知能力提升 的方向发展, 进入了快速发展阶段。美国国家航空 宇航局研制了用于国际空间站舱外作业的宇航员灵 巧手 Robonaut hand ${ }^{[18]}$, 如图 6d 所示。由 1 个手腕 和 5 个手指组成, 共 14 个自由度, 腱绳张力传感器 的加入使得整手的运动控制更加准确。德国宇航中 心先后研制成功了 DLR- I 和 DLR- II 灵巧手 ${ }^{[19-20]}$, 如图 6e、6f 所示, 共集成了 25 个传感器, 包括类 似人工皮肤的触觉传感器、关节扭矩传感器、位置 传感器和温度传感器等, 使灵巧手在灵活性和感知 能力方面都有显著提升。日本岐阜大学先后研制了 三代 Gifu hand ${ }^{[21-22]}$, 该系列多指灵巧手的突出特点 在于其分布式的触觉传感器提升了手对于外界物体 整体的触觉感知, 大幅提高了手抓持和操作的成功 率。意大利 IIT 研制的 $\mathrm{iCub}$ 手高度集成了 12 个触 觉传感器, 48 个压力传感器和 17 个位置传感器以 实现灵巧的操作和丰富的感知能力 ${ }^{[23]}$ 。系统集成度 的提高和感知能力的丰富使得多指手向灵巧操作的 方向更进了一步 ${ }^{[24]}$ 。

高度系统集成的灵巧手具有灵活性和功能性的 优势，但是复杂的系统导致了高额的制造成本并且 降低了系统的可靠性和易维护性。因此近 10 年, 多 指灵巧手设计的一个重要方向是简化系统、提高鲁 棒性。欠驱动手 ${ }^{[25]}$ 通过合理的结构设计以少于手指 关节自由度的驱动器控制手的运动, 以降低整只手 的系统复杂度, 同时提高可靠性。耦合连杆是常见 的欠驱动实现方式，以简单的关节联动实现欠驱 动 ${ }^{[26]}$ 。其缺点是手指在抓取操作任务中只能沿着设 计轨迹运动, 因此手只能抓取特定形状的对象目标。

自适应灵巧手是另一种有效的欠驱动实现方 式, 通过对操作对象的自主适用性包络实现抓取, 例如：立命馆大学设计的 Ritsumeikan Hand 通过耦 合走线实现了 2 个驱动器对 15 个关节的驱动 ${ }^{[27]}$; HERI Hand $^{[28]}$, SPRING hand ${ }^{[29]}$, Columbia hand ${ }^{[30]}$, 耶鲁大学的欠驱动手 ${ }^{[31-32]}$ 等都采用了适应性欠驱动 的结构设计实现了稳定的抓取。意大利比萨大学通 过神经理论分析得出了人手操作中的协同理 论 $^{[33]}$, 并以此为基础设计了一个驱动器带动 5 指 20 个自由度的 soft hand ${ }^{[34]}$, 实现了日常生活中人手高 频的抓取动作。此后, 参数拓扑优化和机器学习等 设计方法被运用到了欠驱动手的设计过程中，以提 高欠驱动抓取的适用性和稳定性 ${ }^{[35-36]}$ 。

欠驱动手虽然在一定程度上以较低的成本、简 化的系统实现了高鲁棒性的抓取任务 ${ }^{[37]}$, 但是由于 欠驱动自身特性使得该类手在操作方面的能力受到 
了限制, 这也是机器人手设计中的鲁棒性和功能性 之间辩证取舍的结果。这样的矛盾也促使研究人员从 具体操作过程研究入手, 进行灵巧手的设计与研究 ${ }^{[38]}$ 。 如 MIT 研制的转子灵巧手通过主动驱动滚轮和手指结 合的方式实现了对物体六自由度非完整空间运动的操 作 ${ }^{[39]}$ 。耶鲁大学设计的 Steward 平台并联机械手巧妙 地实现了对物体平移和旋转的操作 ${ }^{[40]}$ 。另外 Piching hand $\mathrm{d}^{[40-41]} 、$ grasper hand $\mathrm{d}^{[42]} 、$ Closed hand $\mathrm{d}^{[43]}$ 都是依据既 定的抓持或操作任务而专门设计的灵巧手, 不能实现 类似人手的普遍抓持和操作能力。
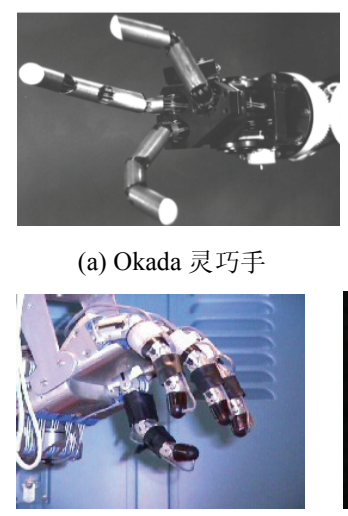

(c) Utah/MIT 灵巧手

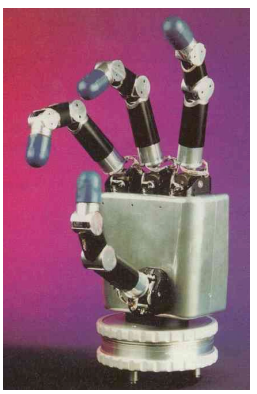

(e) DLR-I

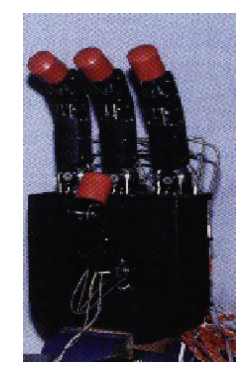

(g) HIT 灵巧手

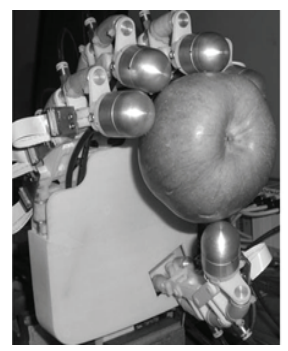

(i) 气动柔性多指手 (a) Okada 灵巧手

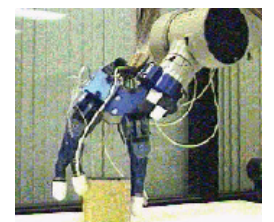

(b) Stanford/JPL 灵巧手

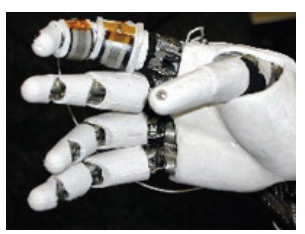

(d) Robonaut hand

(f) DLR- II

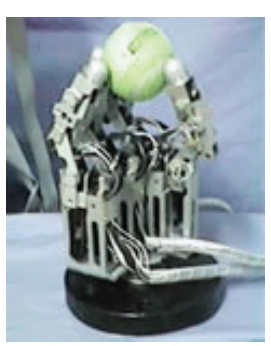

(h) BH-4 灵巧手

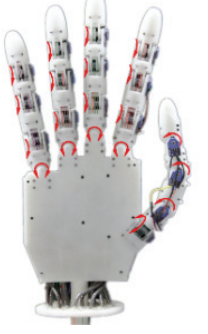

(j) 全驱动多指手

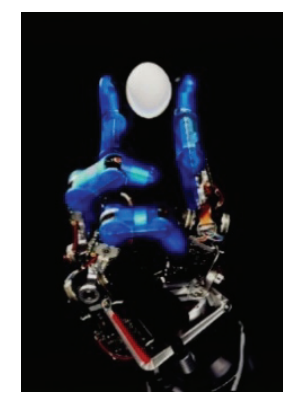

图 6 代表性的多指灵巧手研发
20 世纪 80 年代后期, 国内一些机器人研究机 构和部分高等院校相继开展了机器人多指灵巧手的 研究工作, 其中具有代表性的是哈尔滨工业大学的 HIT 四指仿人灵巧手 ${ }^{[44]}$ 和北京航空航天大学的 $\mathrm{BH}$ 系列多指手 ${ }^{[45-46]}$ 。HIT 手具有 4 个完全相同的手指, 12 个可控自由度, 通过腱传动系统传递运动和力, 如图 $6 \mathrm{~g}$ 所示。BH-4 灵巧手具有 4 指 16 个自由度, 如图 6h 所示。浙江工业大学将气动软体驱动器与刚 性结构进行融合设计、研发了气动柔性多指灵巧

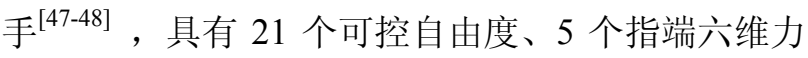
传感器, 并且具有柔性和抓持适应性, 如图 6i 所示。 为了模拟人手的运动灵活性和柔顺性, 浙江工业大 学课题组基于气动肌肉驱动器研发了全驱动无耦合 柔性多指灵巧手 ${ }^{[49]}$, 由气动肌肉驱动和肌腱传动, 具有 25 个独立可控自由度, 如图 6j 所示。

目前已有多家公司制造出商品化的机器人多指 灵巧手。Shadow dexterous hand 是英国 Shadow Robot 公司推出的先进仿人型机器人手 ${ }^{[50]}$, 如图 7a 所示, 拥有 24 个自由度和 20 个可单独控制的自由 度, 并且可配备指端触觉传感器, 是目前最成熟的 商品化多指灵巧手之一。另外, 德国 SCHUNK 的 $\mathrm{SF} 5 \mathrm{H}^{[51-52]}$ 也是商品化的多指灵巧手, 如图 7b 所示, 有 8 个独立控制自由度, 不具备接触等感知功能。 德国 FESTO 公司于 2019 年发布了一款关节结构的 柔性多指灵巧手 Bionic Soft Hand, 如图 7c 所示, 具有 12 个自由度, 可通过强化学习方法模拟人手操 作。北京因时机器人科技有限公司的 RH56BF3 仿 人型多指灵巧手具有 6 个驱动器和 5 个接触力传感 器, 如图 7d 所示。这些产品虽然被命名为多指灵巧 手, 但还是不能实现人手的自由灵活程度和操作能 力, 并且价格昂贵, 推广应用难度较大。

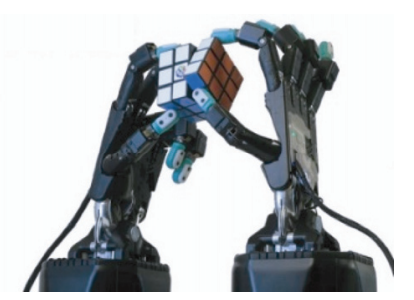

(a) Shadow hand

(c) Bionic Soft Hand

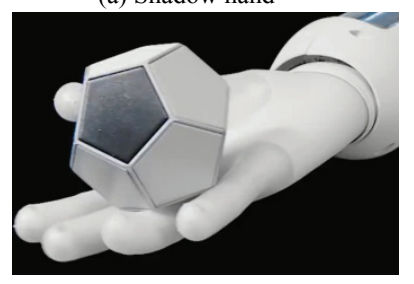

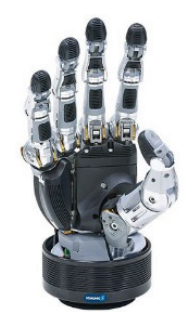

(b) SCHUNK SF5H hand

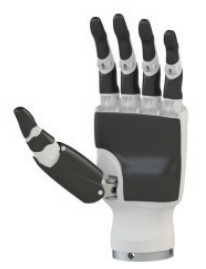

(d) RH56BF3 五指手
图 7 代表性的多指灵巧手产品 
2 多指灵巧手的自身复杂性与用户 易用性

随着机器人应用领域的不断拓展, 其应用场景、 工作对象和任务日趋复杂。对类人手结构和功能的 机器人多指灵巧手的需求与日俱增, 其主要原因可 归纳为三个方面: (1) 复杂多样的操作对象要求机器 人的末端执行器具有类似人手的复杂结构和功能;

(2) 人造世界的各种物品和工具都是以人手的使用 方便为基本原则而设计开发, 所以也只有仿人型机 器人多指灵巧手才是最适合的操作工具; (3) 仿人型 机器人多指灵巧手符合人类的心理认知和日常生活 习惯，容易被市场和用户接受。

\section{1 自身复杂性}

机器人多指灵巧手的自身复杂性主要表现在仿 生结构、驱动、传动、感知、复合材料、建模与控 制等方面的关键技术。

（1）仿生结构: 从手指和手掌的外形上看, 目 前已有的各种多指灵巧手多是模仿了人手的结构和 外观, 具有鲜明的仿生设计特点。然而, 现有的多 指灵巧手的内部结构, 特别是手指关节等运动结构, 主要还是采用传统的机械式铰链转动关节设计。机 械式转动关节的优点是结构稳定可靠、运动可测可 控, 然而同时给驱动传动的设计以局限性, 使其不 得不局限地采用相应的机械式传动, 如齿轮传动等, 使得灵巧手结构几杂而笨重。为了解决上述问题, 有学者采用了模拟动物肌腱的腱绳传动方式, 该方
案在轻量化灵巧手的基础上能够很好地适应传统的 机械结构。但是腱绳在运动过程中会发生非线性的 形变, 同时走线过程中也会引入摩擦问题, 增大了 灵巧手精确控制的难度; 而且, 复杂的走线结构也 增加了本体复杂度和运行维护的难度。

传统机械方式的结构设计虽然外观和形式上实 现了仿生, 但是在运动功能实现上远远达不到人手 的灵活精巧程度和目标适应性。因此, 部分学者尝 试软指手的设计, 软体手在很大程度上提升了抓取 操作的鲁棒性和柔顺性, 但是其指端输出力较小, 并且难以建立软指的精确数学模型、目前仍无法实 现精确的运动和力控制。为了解决软指手刚度过小、 输出力不可控的问题, 采用刚柔结合的结构, 以实 现柔顺性和可靠性的统一。但是仍然存在材料和结 构的有机结合、刚柔特性的融合与取舍等问题。

已有学者进行了灵巧手的内部结构仿人化设 计, 如 FAUDZI 等人研制的 HR-Hand 以 Mckibben 气动肌肉为驱动来模仿人类肌肉。华盛顿大学通过 仿生设计重现了人手上的韧带特征实现了人手分离 式的关节设计, 实现了灵巧手拟人化的运动。特别 是韩国技术教育大学基于关节生物解剖结构设计的 仿人手指 ${ }^{[53-54]}$, 其关节结构、运动方式、驱动传动 布置乃至润滑方式都极力模仿人手的构造, 实现了 良好的运动灵活性, 为机器人多指灵巧手的深度仿 生研究开辟了一个崭新的思路。

上述各种灵巧手的结构方案如表 1 所示。

表 1 现有灵巧手结构设计方案

\begin{tabular}{|c|c|c|c|}
\hline 仿生结构设计类别 & 优点 & 缺点 & 案例 \\
\hline 机械式铰链设计 ${ }^{[52,55-56]}$ & $\begin{array}{l}\text { 传动效率高, 响应快, 关节刚度高, } \\
\text { 输出力稳定且可控。 }\end{array}$ & $\begin{array}{l}\text { 结构冗杂, 笨重, 柔性不足, 抗冲击性 } \\
\text { 能较弱, 对手内空间配置要求较高。 }\end{array}$ & \\
\hline $\begin{array}{l}\text { 腱绳拮抗驱动设 } \\
\text { 计 }^{[31,49,57]}\end{array}$ & $\begin{array}{l}\text { 轻量化, 柔顺适用性, 与传统结构 } \\
\text { 相适应。 }\end{array}$ & $\begin{array}{l}\text { 关节刚度受腱绳形变影响, 腱绳与手指 } \\
\text { 结构摩擦干扰大, 传动效率低, 寿命较低, } \\
\text { 维修成本高。 }\end{array}$ & \\
\hline 软指手 ${ }^{[58-60]}$ & $\begin{array}{l}\text { 结构柔性高, 交互安全, 成本低廉, } \\
\text { 抗干扰。 }\end{array}$ & $\begin{array}{l}\text { 输出力小, 结构刚度低, 建模困难, 控 } \\
\text { 制精度低。 }\end{array}$ & \\
\hline 刚柔混合结构 ${ }^{[61-63]}$ & 柔顺，可靠。 & $\begin{array}{l}\text { 刚柔材料和结构的融合设计困难, 刚性 } \\
\text { 与柔性的跨量级建模困难。 }\end{array}$ & \\
\hline 特殊构型 ${ }^{[64-66]}$ & 多功能, 成本低。 & $\begin{array}{l}\text { 通用性差, 结构与普通灵巧手差异大, } \\
\text { 使用学习成本高。 }\end{array}$ & \\
\hline 仿生生物关节 ${ }^{[53,67-68]}$ & 高度仿人化, 柔顺, 抗冲击, 可控。 & 成本高, 系统复杂, 对驱动器要求高。 & \\
\hline
\end{tabular}


(2) 驱动器: 机器人多指灵巧手的驱动器主要 包括电机、气缸、液压缸、人工肌肉以及近年来涌 现的各种新型智能驱动器 ${ }^{[69]}$ 。电机是一直以来最为 普遍采用的驱动方式, 其标准化、稳定可靠、精度 高、响应快、驱控一体等特点不言而喻; 然而也存 在质量体积大、成本高等缺点, 给仿人型的多指灵 巧手结构设计和推广应用造成一定的困难。故常用 的措施是将驱动电机集中设计在手掌空间内或手臂 内部, 其运动和动力通过腱绳等方式远程传递到各 个手指的关节。这样的布局又使得腱绳的空间走线 变得复杂, 摩擦、腱绳的非线性伸缩变形等问题随 之而来, 给建模和控制带来困难。

气缸或液压缸驱动方式主要应用于需要较大输 出力的场合 ${ }^{[70-71]}$ 。气缸由于其难以实现精确的位置 控制, 常用在简单的抓持手, 不能实现多关节的灵 活运动。人手的质量只占体重的 $1.2 \% \sim 1.8 \%$, 但是 可以实现单手吊环引体等大力输出动作, 就目前的 各种驱动技术而言, 液压驱动能够实现大力抓持的 可能性最大。然而液压系统庞大, 需要在小型化、 便携性等方面的进一步研究。

人工肌肉及各种新型智能驱动器近年来被广泛 应用于多指灵巧手的研发。特别是采用气动人工肌 肉的 Shadow dexterous hand 已经产品化, 证明了人 工肌肉等新型驱动器的应用可行性。即便如此, 庞 大的结构尺寸、数学建模困难、响应带宽小、控制 精度低等问题依然是人工肌肉在应用中面临的、但 长久以来尚未得到有效改善的问题 ${ }^{[72-73]}$ 。气动人工 肌肉的小型化、微型化研究 ${ }^{[54,74]}$ 有望解决应用中结 构尺寸庞大的问题。各种智能驱动器如 SMA、DEA、 IPMC 等 ${ }^{[75-76]}$ 仍然处于功能试验阶段, 在激励源、 驱动能力、响应特性等诸多方面还不能满足多指灵 巧手的驱动要求。

(3) 传动方式: 早期的多指灵巧手的手指内曾 采用齿轮、连杆等传动机构。然而因为尺寸和质量 大, 运动不灵活等问题逐渐被淘汰 ${ }^{[77]}$ 。模仿动物肌 腱传动方式的腱绳传动是后来被各种多指灵巧手设 计所广泛采用的传动方式。腱绳可以根据需要采用 各种材料、与腱鞘或套管配合在手臂、手掌和手指 内部灵活布局走线; 并且腱绳一般都具有一定的弹 性, 为手指运动提供了一定程度的柔顺性和抓持适 应性。然而, 腱绳传动需要滑轮或绕线机构以实现 其收放, 又会造成结构尺寸增大。柔性扭绞腱绳 ${ }^{[78-79]}$ 是一种独特的传动方式, 利用柔性腱绳在扭绞过程 中的缩短效应, 实现运动和动力的同步传递, 且无 须额外机械结构, 大大简化了结构设计和尺寸空间。
利用渔线加热扭转旋绕复合变形 ${ }^{[80-81]}$ 产生驱动输出 也是一种集驱动与传动于一身的新传动方式。

(4) 感知技术: 机器人多指灵巧手的感知技术 可以分为内部感知和外部感知 ${ }^{[82]}$ 。与传统机器人类 似, 内部感知主要检测关节位置、速度和加速度等 运动参数并反馈至控制器。对于具有明确机械转动 或移动形式的关节，检测其运动参数的技术和传感 器已经臻于成熟, 在应用于手指结构时需要解决小 型化问题。相比较而言, 外部感知的信息种类较多、 来源和特性复杂 ${ }^{[83]}$ (如与外界的作用力、触觉、温度、 湿度等), 难以实现, 是机器人多指灵巧手感知复杂 性的主要方面。由于仿生手指表面的软性材料及曲 面结构、与外界接触过程中的交互作用与复杂变形 等情况, 常规的传感器在结构、安装方式、检测能 力和适应性方面都远远达不到要求, 因此进行类人 皮肤及神经系统的感知技术及器件研发得到广泛的 关注, 电子皮肤 ${ }^{[84-86]}$ 将是最理想的多指灵巧手感知 系统并且已经成为一个热点研究方向。

(5) 复合/智能材料: 除了轻量化、高强度等常 规使用性能要求外, 机器人多指灵巧手对于复合材 料乃至智能材料的需求, 更主要的体现在特殊的关 节结构设计、新型驱动和传动技术、感知和自感知 技术。例如仿人型关节设计中必须考虑刚、柔、软、 液的综合实现以及运行过程耦合, 在同一个结构上 采用不同的材料或者复合材料以同时满足上述特性 和运动的需求。如前所述, 近年来涌现的各类新型 驱动器和传动技术, 更是严重依赖于新型智能复合 材料, 如将软性材料与导电材料 ${ }^{[87]}$ 、燃料 ${ }^{[88]}$ 、电/ 磁流变材料 ${ }^{[89]}$ 等的融合。与之类似, 电子皮肤等新 型智能感知技术的发展也同样与复合材料的发展紧 密相关。甚而至于出现了将驱动、传动、感知进行 一体化设计的新研发方向 ${ }^{[85]}$, 这更离不开复合、智 能材料技术的支撑 ${ }^{[00]}$ 。

(6) 数学模型与控制: 机械物理系统的运行控 制高度依赖于对其本质规律的掌握及其数学描述, 数学模型的准确性决定相应的控制策略选择、控制 方法的设计、过程和控制参数获取及调整算法。机 器人多指灵巧手的数学模型主要有两个层面, 即: 本体机械物理模型和目标操作理论模型。

在多指灵巧手本体机械物理建模层面, 日益复 杂的机械结构, 特别是具有柔性、软体、甚至液态 材料的结构, 使得准确建模异常困难。另外一个思 路是避开机械本体的本质问题讨论, 采用实验测试、 数据分析、深度学习的方法建立多指灵巧手的辨识 模型, 在实验样本范围内可以建立较为理想的计算 
模型, 但对于实验未覆盖的情况, 这种方法的泛化 能力还有待提高。

在目标操作理论, 特别是模仿人手的操作过程 仿生建模层面, 从 20 世纪 80 年代到 21 世纪初期曾 经有一次研究热潮 ${ }^{[11-92]}$, 特别是北京航空航天大学 机器人研究所在张启先院士带领下进行了系统深入 的研究工作, 取得了一系列成果 ${ }^{[93]}$ 。这一阶段的研 究主要基于力学和几何原理研究多指灵巧手的有效 抓持及其稳定性问题, 极少涉及目标物体的操作问 题 ${ }^{[94]}$ 。随着大数据、深度学习等人工智能技术的新 一轮热潮涌现, 以及应用领域对多指灵巧手的需求 凸显, 近年来更多的研究人员进一步思考机器人多 指灵巧手对人手的操作能力的学习和复现问题, 因 此涌现出大量的基于深度学习的人手操作过程辨 识、建模和机器人多指灵巧手控制研究 ${ }^{[95-98]}$, 大大 简化了建模过程及其难度。然而与上述本体机械物 理建模存在一样的难题, 即对于超出试验样本的适 用性和泛化能力有限。即便是与试验同样的操作对 象和任务, 目前得到的模型和控制方法也无法实现 与人手相媲美的灵活操作控制 ${ }^{[99]}$ 。所以我们认为, 从人手的目标操作过程分析入手, 抽象并建立基本 操作元模型库, 进一步建立操作过程规划规则和方 法, 是进行机器人多指灵巧手建模研究的基本方向, 当然也可以借助于人工智能方法强大的数据分析和 学习能力, 取长补短, 在建模复杂性、准确性、适 应性和泛化能力上取得综合性的提高。

\section{2 应用需求的复杂性}

从仿生应用的复杂性角度看, 机器人多指灵巧 手可以分为三个层次, 即: 部分功能复现、灵巧操 作功能复现和人手功能的增强。

(1) 部分功能复现: 主要应用于康复训练、运 动助力等场合, 如手指运动功能康复机器人、助力 机械手、假肢手等 ${ }^{[100-101]}$ 。这类应用一般只需要复 现人手的部分运动功能, 对患者进行运动能力的恢 复、辅助或替代, 并且对模型准确性及控制精度要 求相对不高。

(2) 灵巧操作功能复现: 这一类应用预期需求 最广泛, 特别是生活社交、电商物流、食品加工流 转、甚至工业操作与装配领域已经提出了强烈的需 求。这类应用也是对机器人多指灵巧手具有人手结 构和功能的期望最高的。传统的固定工位和工艺流 程的工业流水线不能满足当前个性化、小批量、短 周期的产品生产需要, 而模拟人类操作的双臂人形 机器人是实现智能化、柔性化生产的最佳工具, 其 中拟人结构和功能的多指灵巧手是最关键的部件之
一。在电商物流和食品加工等应用中的分类分拣、 抓取辨识等操作同样需要灵活功能的机械手。尤其 是在日常生活和社交机器人应用中, 由于人造环境 和物品的人手适用性特点、人类对类人型机器人和 机械手的心理认同等因素, 需要机器人多指灵巧手 具有人手的结构、外形, 并且能够处理日常生活中 的各种目标物体和动作任务, 因此对其复杂操作功 能要求极高。

（3）人手功能增强: 虽然机器人多指灵巧手目 前还远远不能达到人手的结构精巧性和操作动作灵 活性, 但是随着材料、驱动、控制、人工智能等技 术的发展和科研人员的不解努力, 终究会在某些方 面逼近乃至超越人手, 例如更大的输出力/自重比、 更复杂的结构、更多的自由度、更灵活的操作能力、 更广泛的目标及环境适应性等。

\section{3 基于多指灵巧手的复杂应用简化实现}

在工业应用领域, 传统的自动化系统是针对具 体的产品进行工艺路线设计并开发分工位、固定式 生产线, 这是实现机器换人、减员增效、提高效率 和产品质量的有效方法, 特别适用于大批量生产模 式。但是当前和未来市场需求日趋个性化, 短周期 小批量定制化生产逐渐成为主流。在这样的前提下, 传统工业自动化系统由于设计周期长、人力资金投 入大、复用性差、调度重组的柔性弱、任务执行灵 活性低等原因而不再适用, 基于机器人的智能制造 成为企业关注的焦点。机器人多指灵巧手在同一个 工位即可完成传统自动化流水线多个甚至十多个工 位才能完成的加工或装配工艺。并且由于具有配套 的离线编程系统, 可以根据不同的订单任务, 灵活 便捷地实现对机器人多指灵巧手操作的再规划编程 和直接控制。实现了工业生产中换单换线生产的轻 量化设计和简单实现, 运行过程、使用维护简单快 捷, 并且一次投入可重复使用, 将极大地节约运行 和维护成本。

与工业应用一样, 机器人多指灵巧手在康复训 练、生活社交等领域的应用有异曲同工之妙, 将会 避免传统机械系统的繁冗结构、任务分析、动作分 解、分布控制等工作, 直接采用仿人的工作方式完 成相应的工作内容, 使得应用过程就像人手的使用 一样自然顺畅。

可见, 机器人多指灵巧手系统本身的研发虽然 复杂、困难重重, 但研发成功以后, 即可实现类人 的各种操作功能和执行能力, 将在工业、生活服务 等各个领域得到推广并降低应用成本、简化应用 难度。 


\section{3 多指灵巧手本体研究的趋势与挑战}

虽然应用需求凸显并日趋旺盛, 这些需求也引 领着机器人多指灵巧手的研发方向和发展趋势, 但 目前的技术和产品依然存在诸多问题和挑战, 亟待 解决。

(1) 深度仿生: 如前所述, 多指灵巧手最初是 从结构, 特别是外形上进行仿生设计。随着更多更 复杂的应用功能需求提出, 研究人员逐渐开始从人 手内在结构、驱动和传动原理等方面思考其仿生结 构的设计, 并逐步延伸到复合材料、智能材料的研 制和设计, 智能材料的研究又反过来影响多指灵巧 手的仿生设计; 同时, 还应该注意到, 对于人手操 作过程的研究, 也应属于机器人多指灵巧手仿生研 究的范畴, 并且是非常重要的一个方面, 会在很大 程度上决定多指灵巧手的仿生程度并成为一个重要 的衡量指标。

（2）柔性感知技术: 由于手部结构精巧复杂、 功能多样、感觉丰富, 实现仿生的机器人多指灵巧 手必然需要像人类皮肤一样能够感知丰富的多源信 息的柔性感知技术和传感器。然而, 电子皮肤的研 发涉及材料、机械、信息、人工智能乃至生物等多 个学科领域。人类皮肤的生物感知机理及其复现方 法尚不明确, 实现类人皮肤的复杂、柔性结构并保 持优良的信息采集与处理技术依然任重而道远。

(3) 操作过程规划与控制策略: 实现仿人的灵 巧操作能力是机器人多指灵巧手研发的重要目标。 然而, 目前对于人手的灵巧操作过程的基本原理、 规划方法尚不明确。虽然众多实验室采用机器视觉 和深度学习相结合的方法进行了特定动作或任务的 学习建模, 但是鲜见人手操作过程的基本原理分析 研究。从基本原理和规则角度剖析人手的灵巧操作 过程, 并运用数学的方法加以描述, 更具有科学性 和通用性, 是进行机器人多指灵巧手灵巧操作规划 与控制的理论基础。

(4) 成本控制: 现有的机器人多指灵巧手的销 售价格普遍奇高, 例如: 哈尔滨工业大学-德国宇航 中心合作开发的 HIT/DLR 灵巧手售价在 90 万元人 民币以上，Shadow Dexterous Hand 报价约 30 万美 元, 德国 SCHUNK 公司的 SVH 五指手报价 60 万 元人民币以上。高昂的价格是推广应用一大障碍, 上述三种多指手产品也仅仅在科研实验室里用于科 学研究和应用基础开发, 尚未发现有商业或工业化 应用的报道。近年来, 各类科研机构开始投入研发
低成本的机器人多指手, 从材料、加工方式、驱动 器件、感知器件等方面进行低成本化设计和制造, 开发了一些样机, 但其灵活性、感知丰富性和可靠 性等指标普遍较低。所以，如何在性能和成本之间 取得合理的平衡也是值得研究的课题。

\section{4 结论}

有关机器人多指灵巧手的研究工作已经有半个 世纪之久, 科研机构和相关的公司也研制出了几款 具有代表性的多指灵巧手样机和产品, 但是于实际 应用还有相当大的差距。随着经济社会的高速发展, 从产业界的智能制造、健康领域的智慧医疗到社会 领域的智慧城市、智慧生活, 各行各业都对机器人 提出了更高更广更深的需求，同样对拟人化的多指 灵巧手也提出了迫切的需求和期待。

为满足社会和市场对灵活、精巧、低价的需求, 机器人多指灵巧手研究在仿生机构、驱动、传动、 感知、复合/智能材料、建模、控制等方面得以持续 不断的深入, 也已取得了一些阶段性的进步和成果, 但依然不足以形成满足社会需求的产品。在深度仿 生、柔性感知、操作规划和控制策略、成本控制等 方面依然存在巨大的困难和挑战, 这也是近年来各 国科研人员聚焦研发的重点和热点方向。

机器人多指灵巧手的本体、建模、感知、规划 与控制的复杂性, 源于人类社会对它的拟人化灵活 操作能力预期。人们在使用自己的双手执行生活和 生产中的各种操作任务时, 常常是潜意识中即完成 了目标任务的理解、分解和手指协同工作的过程规 划与控制。这些看似习以为常, 简单至极的工作能 力是人类经历数百万年进化才具有的, 因此研制复 现人手功能和能力的机器人多指灵巧手必然也将经 历艰难、复杂、曲折的过程。不过, 一旦机器人多 指灵巧手具有了人手的灵活操作能力, 即便是部分 功能、抑或是部分场景、有限环境下的灵活应用能 力, 都将极大地简化相关领域的开发工作、缩短研 发周期、降低使用难度、节约研发和运维成本。

总而言之, 机器人多指灵巧手从简单结构发展 到现在努力追求的繁复系统, 其目的是为了将现有 的繁杂的操作类工作及其相关技术装备进行简化而 实现之。可以预期, 最终的结果也必然是这样。

\section{参 考 文 献}

[1] 刘辛军, 于靖军, 王国彪, 等. 机器人研究进展与科学 挑战[J]. 中国科学基金，2016，30(5)：425-431. 
LIU Xinjun, YU Jingjun, WANG Guobiao, et al. Research trend and scientific challenges of robotics[J]. China Science Foundation, 2016， 30(5): 425-431.

[2] DING Han, YANG Xuejun, ZHENG Nanning, et al. Tri-co robot: A Chinese robotic research initiative for enhanced robot interaction capabilities[J]. National Science Review，2018，5(6): 799-801.

[3] PIAZZA C, GRIOLI G, CATALANO M G, et al. A century of robotic hands[J]. Annual Review of Control Robotics and Autonomous Systems, 2019，2(1): 1-32.

[4] 张进华, 王蹈, 洪军, 等. 软体机械手研究综述 [J]. 机 械工程学报, 2017, 53(13): 19-28.

ZHANG Jinhua, WANG Tao, HONG Jun, et al. Review of soft-bodied manipulator[J]. Journal of mechanical engineering, 2017, 53(13): 19-28.

[5] 宗光华, 刘海波, 程君实. 机器人技术手册[M]. 北京: 科学出版社, 1996.

ZONG Guanghua, LIU Haibo, CHENG Junshi. Robotics manual[M]. Beijing: Science Press, 1996.

[6] NIE K, WAN W, HARADA K. A hand combining two simple grippers to pick up and arrange objects for assembly[J]. IEEE Robotics and Automation Letters, 2019, 4(2): 958-965.

[7] HASAN M R, VEPA R J, SHAHEED H, et al. Modelling and control of the Barrett hand for grasping[C]// UKSim-Amss 15th International Conference on Computer Modelling and Simulation, Cambridge, UK, IEEE, 2013: 230-235.

[8] SUAREZ-RUIZ F, GALIANA I, TENZER Y, et al. Grasp mapping between a 3-finger haptic device and a robotic hand[C]// 9th International Conference on Human Haptic Sensing and Touch Enabled Computer Applications, Berlin, Heidelberg, Springer, 2014, 8618: 275-283.

[9] SUZUMORI K, IIKURA S, TANAKA H. Applying a flexible microactuator to robotic mechanisms[J]. IEEE Control Systems Magazine, 1992, 12(1): 21-27.

[10] 鲍官军, 张水波, 陈亮, 等. 基于气动柔性驱动器的球 果采摘末端抓持器[J]. 农业机械学报, 2013，44(5): 242-246.

BAO Guanjun, ZHANG Shuibo, CHEN Liang, et al. Design of spherical fruit end-grasper based on FPA[J]. Journal of Agricultural Machinery, 2013, 44 (5): 242-246.

[11] BAO Guanjun, MA Xiaolong, LUO Xingyuan, et al. Full compliant continuum robotic finger and its kinematic model[J]. Iranian Journal of Science and
Technology-Transactions of Mechanical Engineering, 2014, 38(M2): 389-402.

[12] WANG Zhoukui, OR K, HIRAI S. A dual-mode soft gripper for food packaging[J]. Robotics and Autonomous Systems, 2020, 125: 103427.

[13] BAO Guanjun, FANG Hui, CHEN Lingfeng, et al. Soft robotics: Academic insights and perspectives through bibliometric analysis[J]. Soft Robotics, 2018, 5(3): 229-241

[14] ZHAO Huichan, O’BRIEN K, LI Shuo, et al. Optoelectronically innervated soft prosthetic hand via stretchable optical waveguides[J]. Science Robotics, 2016, 1(1): eaai7529.

[15] TOKUJI O. Computer control of multijointed finger system for precise object-handling[J]. IEEE Transactions on Systems, Man, and Cybernetics, 1982, 12(3): 289-299.

[16] MATTHEW T M, J.KENNETH S, JOEY K P. Robot hands and the mechanics of manipulation[J]. MIT Press: Journal of Dynamic Systems, Measurement, and Control, 1985.

[17] JACOBSEN S C, WOOD J E, KNUTTI D F, et al. The UTAH/MIT dextrous hand: Work in progress[J]. The International Journal of Robotics Research， 1984，3(4): 21-50.

[18] LOVCHIK C S, DIFTLER M A. The robonaut hand: A dexterous robot hand for space[C]// 1999 IEEE International Conference on Robotics and Automation (Cat. No. 99CH36288C), Detroit, MI, USA, IEEE, 1999: 907-912.

[19] HIRZINGER G, FISCHER M, BRUNNER B, et al. Advances in robotics : The DLR experience[J]. International Journal of Robotics Research, 1999, 18(11): 1064-1087.

[20] LIU Hong, Wu K, MEUSEL P, et al. Multisensory five-finger dexterous hand: The DLR/HIT Hand II[C]// 2008 IEEE/RSJ International Conference on Intelligent Robots and Systems, Nice, France, IEEE, 2008 : 3692-3697.

[21] MOURI T, KAWASAKI H, YOSHIKAWA K, et al. Anthropomorphic robot hand: Gifu hand III[J]. JRSJ, 2002: 1288-1293.

[22] KAWASAKI $\mathrm{H}$, KOMATSU $\mathrm{T}$, UCHIYAMA K. Dexterous anthropomorphic robot hand with distributed tactile sensor: Gifu hand II[J]. IEEE-ASME Transactions on Mechatronics, 2002, 7(3): 296-303. 
[23] SCHMITZ A, PATTACINI U, NORI F, et al. Design, realization and sensorization of the dexterous $\mathrm{iCub}$ hand[C]// IEEE-RAS International Conference on Humanoid Robots, Nashville, TN, USA , IEEE, 2010: 186-191.

[24] LI Miao, HANG Kaiyu, KRAGIC D, et al. Dexterous grasping under shape uncertainty[J]. Robotics and Autonomous Systems, 2016, 75: 352-364.

[25] BIRGLEN L, LALIBERTE T, GOSSELIN C M. Underactuated robotic hands[M]. Berlin Heidelberg: Springer, 2007.

[26] YUN Y, AGARWAL P, FOX J, et al. Accurate torque control of finger joints with UT hand exoskeleton through Bowden cable SEA[C]// IEEE/RSJ International Conference on Intelligent Robots and Systems, Daejeon, South Korea, IEEE, 2016: 390-397.

[27] MITSUI K, OZAWA R, KOU T. An under-actuated robotic hand for multiple grasps[C]// IEEE/RSJ International Conference on Intelligent Robots and Systems, Tokyo, Japan, IEEE, 2013: 5475-5480.

[28] REN Zeyu, ZHOU Chengxu, XIN Songyan, et al. Heri hand: A quasi dexterous and powerful hand with asymmetrical finger dimensions and under actuation[C]// IEEE/RSJ International Conference on Intelligent Robots and Systems, Vancouver, BC, Canada, IEEE, 2017: 322-328.

[29] CARROZZA M C, SUPPO C, SEBASTIANI F, et al. The SPRING hand: Development of a self-adaptive prosthesis for restoring natural grasping[J]. Autonomous Robots, 2004, 16(2): 125-141.

[30] WANG Long, DELPRETO J, BHATTACHARYYA S, et al. A highly-underactuated robotic hand with force and joint angle sensors[C]// 2011 IEEE/RSJ International Conference on Intelligent Robots and Systems, San Francisco, CA, USA, IEEE, 2011: 1380-1385.

[31] WISTE T, GOLDFARB M. Design of a simplified compliant anthropomorphic robot hand[J]. 2017 IEEE International Conference on Robotics and Automation, 2017(1): 3433-3438.

[32] DOLLAR A M, HOWE R D. The highly adaptive SDM hand: Design and performance evaluation[J]. International Journal of Robotics Research， 2010， 29(5): 585-597.

[33] RENDA F, GIORELLI M, CALISTI M, et al. Dynamic model of a multibending soft robot arm driven by cables[J]. IEEE Transactions on Robotics, 2014，30(5):
1109-1122.

[34] CATAlano M G, GRioli G, FARNiOli E, et al. Adaptive synergies for the design and control of the Pisa/IIT SoftHand[J]. The International Journal of Robotics Research, 2014, 33(5): 768-782.

[35] ZHANG Hongying, KUMAR A S, FUH J Y H, et al. Topology optimized design, fabrication and evaluation of a multimaterial soft gripper[C]// 2018 IEEE International Conference on Soft Robotics, Livorno, Italy, IEEE, 2018: 424-430.

[36] ZHANG Hongying, KUMAR A S, FUH J Y H, et al. Topology optimized multimaterial soft fingers for applications on grippers, rehabilitation, and artificial hands[J]. IEEE/ASME Transactions on Mechatronics, 2019, 24(1): 120-131.

[37] DEIMEL R, BROCK O. A novel type of compliant and underactuated robotic hand for dexterous grasping $[\mathrm{J}]$. International Journal of Robotics Research, 2016, 35(1-3): 161-185.

[38] CUI Lei, SUN Jie, DAI J S. In-hand forward and inverse kinematics with rolling contact[J]. Robotica, 2017, 35(12): 2381-2399.

[39] YUAN Shenli, EPPS A D, NOWAK J B, et al. Design of a roller-based dexterous hand for object grasping and within-hand manipulation[C]// 2020 IEEE International Conference on Robotics and Automation, Paris, France, IEEE, 2020: 8870-8876.

[40] MCCANN C M, DOLLAR A M. Design of a stewart platform-inspired dexterous hand for 6-DOF within-hand manipulation[C]// 2017 IEEE/RSJ International Conference on Intelligent Robots and Systems , Vancouver, BC, Canada, IEEE, 2017: 1158-1163.

[41] HASEGAWA S, WADA K, NIITANI Y, et al. A three-fingered hand with a suction gripping system for picking various objects in cluttered narrow space[C]// 2017 IEEE/RSJ International Conference on Intelligent Robots and Systems, Vancouver, BC, Canada, IEEE, 2017: 1164-1171.

[42] GOVINDAN $\mathrm{N}$, KOVVALI $\mathrm{S} \quad \mathrm{S} \quad \mathrm{V}$, CHANDRASEKARAN $\mathrm{K}$, et al. GraspMan - a novel robotic platform with grasping, manipulation, and multimodal locomotion capability[C]// 2018 IEEE International Conference on Robotics and Automation , Brisbane, Australia, IEEE, 2018: 7354-7359.

[43] PEDRO P, ANANDA C, RAFAEL P B, et al. Closed 
structure soft robotic gripper[C]// 2018 IEEE International Conference on Soft Robotics, Livorno, Italy, IEEE, 2018: 66-70.

[44] 何平, 金明河, 刘宏, 等. 机器人多指灵巧手基关节力 矩/位置控制系统的研究 [J]. 机器人, 2002, 24(4): 314-318.

HE Ping, JIN Minghe, LIU Hong, et al. Study of position and torque feedback control system of multi-fingered dexterous robot hand[J]. Robotics, 2002, 24 (4): 314-318.

[45] 尚喜生, 郭卫东, 张浩, 等. BH-4 灵巧手抓持规划与 实现 $[\mathrm{C}] / /$ “面向新世纪中国机器人产业化发展论坛”大 会, 青岛, 2000：196-200.

SHANG Xisheng, GUO Weidong, ZHANG Hao, et al. Planning and realization of $\mathrm{BH}-4$ dexterous hand grip[C]// Conference on "China robot industrialization development forum for the new century", Qingdao, 2000: 196-200.

[46] 王国庆, 张启先, 李大寨, 等. 基于抓持稳定度的多指 灵巧手抓持控制[J]. 航空学报, 1997(3): 294-298.

WANG Guoqing, ZHANG Qixian, LI Dazhai, et al. Grasping control of the dexterous hand based on the degress of stability of grasping [J]. Acta Aeronautica Sinica, 1997(3): 294-298.

[47] 王志恒, 钱少明, 杨庆华, 等. 气动机器人多指灵巧手 —ZJUT Hand[J]. 机器人, 2012, 34(2): 223-230.

WANG Zhiheng, QIAN Shaoming, YANG Qinghua, et al. Multi fingered dexterous pneumatic robot hand[J]. Robotics, 2012, 34(2): 223-230.

[48] WANG Zhiheng, ZHANG Libin, BAO Guanjun, et al. Pneumatic robot multi-fingered dexterous hand-ZJUT hand[J]. Journal of Central South University, 2011, 18(4): 1105-1114.

[49] ZHU Liyao, WANG Wenbiao, TAO Zhicheng, et al. Full-drive decoupled bionic finger : Structure and experimental trials[C]// 2019 IEEE International Conference on Robotics and Biomimetics, Dali, China, IEEE, 2019: 497-502.

[50] LI Shuang, MA Xiaojian, LIANG Hongzhuo, et al. Vision-based teleoperation of shadow dexterous hand using end-to-end deep neural network[C]// 2019 International Conference on Robotics and Automation, Montreal, Canada, IEEE, 2019: 416-422.

[51] ARMSTRONGHELOUVRY B, DUPONT P, DEWIT C C. A survey of models, analysis tools and compensation methods for the control of machines with friction[J].
Automatica, 1994, 30(7): 1083-1138.

[52] CERUlo I, FICUCIEllo F, LiPPiEllo V, et al. Teleoperation of the SCHUNK S5FH under-actuated anthropomorphic hand using human hand motion tracking[J]. Robotics and Autonomous Systems, 2017, 89: $75-84$.

[53] KIM Y, YOON J, SIM Y. Fluid lubricated dexterous finger mechanism for human-like impact absorbing capability[J]. IEEE Robotics and Automation Letters, 2019, 4(4): 3971-3978.

[54] KURUMAYA S, NABAE H, ENDO G, et al. Design of thin McKibben muscle and multifilament structure[J]. Sensors and Actuators A-Physical, 2017, 261: 66-74.

[55] LEE D H, PARK J H, PARK S W, et al. KITECH-Hand: A highly dexterous and modularized robotic hand[J]. IEEE/ASME Transactions on Mechatronics, 2017, 22(2): 876-887.

[56] VAN DER NIET OTR O, REINDERS-MESSELINK H $\mathrm{A}$, BONGERS R M, et al. The i-LIMB hand and the DMC plus hand compared: A case report[J]. Prosthetics and Orthotics International，2010，34(2): 216-220.

[57] MELCHIORRI C, PALLI G, BERSELli G, et al. Development of the UB Hand IV: Overview of design solutions and enabling technologies[J]. IEEE Robotics and Automation Magazine, 2013, 20(3): 72-81.

[58] ZHAO Huichan, O'BRIEN K, LI Shuo, et al. Optoelectronically innervated soft prosthetic hand via stretchable optical waveguides[J]. Science Robotics, 2016, 1(1): eaai7529.

[59] SHE Yu, CHEN Ji, SHI Hongliang, et al. Modeling and validation of a novel bending actuator for soft robotics applications[J]. Soft Robotics, 2016，3(2): 71-81.

[60] TERRYN S, BRANCART J, LEFEBER D, et al. Self-healing soft pneumatic robots[J]. Science Robotics, 2017, 2(9): eaan4268.

[61] ZHOU Jianshu, CHEN Xiaojiao, LI Jing, et al. A soft robotic approach to robust and dexterous grasping[C]// 2018 IEEE International Conference on Soft Robotics, Livorno, Italy, IEEE, 2018: 412-417.

[62] LIU Yuan, JIANG Li, FAN Shaowei, et al. A novel actuation configuration of robotic hand and the mechanical implementation via postural synergies[C]// 2017 IEEE International Conference on Robotics and Automation, Singapore, IEEE, 2017: 2215-2222.

[63] MARTENS M, BOBLAN I. Modeling the static force of a 
Festo pneumatic muscle actuator: A new approach and a comparison to existing models[J]. Actuators, 2017, 6(4): 33.

[64] MA R R, ROJAS N, DOLLAR A M. Spherical hands: Toward underactuated, in-hand manipulation invariant to object size and grasp location[J]. Journal of Mechanisms and Robotics-Transaction of the Same, 2016, 8(6): 061021.

[65] SCHALER E W, RUFFATTO D I, GLICK P, et al. An electrostatic gripper for flexible objects[C]// 2017 IEEE/RSJ International Conference on Intelligent Robots and Systems, Vancouver, BC, IEEE, 2017: 1172-1179.

[66] YUAN Shenli, SHAO Lin, YAKO C L, et al. Design and control of roller grasper V2 for in-hand manipulation[J]. ArXiv, 2020(4): 1.

[67] KIM Y J, YOON J, SIM Y W. Fluid lubricated dexterous finger mechanism for human-like impact absorbing capability[J]. IEEE Robotics and Automation Letters, 2019, 4(4): 3971-3978.

[68] FAUDZI A A M, OOGA J, GOTO T, et al. Index finger of a human-like robotic hand using thin soft muscles[J]. IEEE Robotics and Automation Letters, 2018, 3(1): 92-99.

[69] VANDERBORGHT B, ALBU-SCHAEFFER A, BICCHI $\mathrm{A}$, et al. Variable impedance actuators: A review[J]. Robotics and Autonomous Systems, 2013, 61(12): 1601-1614.

[70] KO T, KAMINAGA H, NAKAMURA Y. Underactuated four-fingered hand with five electro hydrostatic actuators in cluster[C]// 2017 IEEE International Conference on Robotics and Automation, Singapore, IEEE, 2017: 620-625.

[71] PYLATIUK C, SCHULZ S, KARGOV A, et al. Two multiarticulated hydraulic hand prostheses[J]. Artificial Organs, 2004, 28(11): 980-986.

[72] ZHANG Jun, SHENG Jun, O'NEILL C T, et al. Robotic artificial muscles : Current progress and future perspectives[J]. IEEE Transactions on Robotics, 2019, 35(3): 761-781.

[73] 鲍官军, 张亚琪, 许宗贵, 等. 软体机器人气压驱动结 构研究综述 $[J]$. 高技术通讯, 2019, 29(5): 467-479. BAO Guanjun, ZHANG Yaqi, XU Zonggui, et al. Review on pneumatic-driven structure for soft robot[J]. High Tech-communication, 2019, 29(5): 467-479.

[74] 南卓江, 杨扬, 铃森康一, 等. 基于细径 McKibben 型
气动人工肌肉的仿生手研发 $[\mathrm{J}]$. 机器人, 2018, 40(3): 321-328.

NAN Zhuojiang, YANG Yang, KENICHI S, et al. Development of a bionic hand actuated by thin McKibben pneumatic artificial muscle[J]. Robot, 2018, 40(3): 321-328.

[75] GU Guoying, ZHU Jian, ZHU Limin, et al. A survey on dielectric elastomer actuators for soft robots[J]. Bioinspiration and Biomimetics, 2017, 12(1): 011003.

[76] MOHDLSA W, HUNT A, HOSSEINNIA S H. Sensing and self-sensing actuation methods for ionic polymer metal composite (IPMC): A review[J]. Sensors, 2019, 19(18): 3967.

[77] KASHEF S R, AMINI S, AKBARZADEH A. Robotic hand: A review on linkage-driven finger mechanisms of prosthetic hands and evaluation of the performance criteria[J]. Mechanism and Machine Theory, 2020, 145: 103677.

[78] PALli G, NATALE C, MAY C, et al. Modeling and control of the twisted string actuation system[J]. IEEE-ASME Transactions on Mechatronics, 2013, 18(2): 664-673.

[79] WU Lianjun, DE ANDRADE M J, SAHARAN L K, et al. Compact and low-cost humanoid hand powered by nylon artificial muscles[J]. Bioinspiration and biomimetics, 2017, 12(2): 026004.

[80] TANG Xintian, LI Kai, LIU Yingxiang, et al. A general soft robot module driven by twisted and coiled actuators[J]. Smart Materials and Structures, 2019, 28(3): 035019.

[81] PALli G, HOSSEINI M, MELCHIORRI C. Twisted string actuation with sliding surfaces[C]// 2016 IEEE/RSJ International Conference on Intelligent Robots and Systems, Daejeon, Korea, IEEE, 2016: 260-265.

[82] YOUSEF H, BOUKALLEL M, ALTHOEFER K. Tactile sensing for dexterous in-hand manipulation in roboticsA review[J]. Sensors and Actuators A-Physical, 2011, 167(2): 171-187.

[83] KAPPASSOV Z, CORRALES J A, PERDEREAU V. Tactile sensing in dexterous robot hands - Review[J]. Robotics and Autonomous Systems, 2015, 74: 195-220.

[84] YANG Junchang, MUN J, KWON S Y, et al. Electronic skin: Recent progress and future prospects for skinattachable devices for health monitoring, robotics, and prosthetics[J]. Advanced Materials, 2019, 31(48) : 
1904765.

[85] LI Shuo, BAI Hedan, SHEPHERD R F, et al. Bio-inspired design and additive manufacturing of soft materials, machines, robots, and haptic interfaces $[\mathrm{J}]$. Angewandte Chemie-International Edition , 2019, 58(33) : 11182-11204.

[86] YAMAGUCHI T, KASHIWAGI T, AIRE $\mathrm{T}$, et al. Human-like electronic skin-integrated soft robotic hand[J]. Advanced Intelligent Systems，2019， 1(2): 1900018.

[87] LI Tiefeng, LI Guorui, LIANG Yiming, et al. Fast-moving soft electronic fish[J]. Science Advances, 2017, 3(4): e1602045.

[88] WEHNER M, TRUBY R L, FITZGERALD D J, et al. An integrated design and fabrication strategy for entirely soft, autonomous robots[J]. Nature, 2016，536(7617): 451-455.

[89] MIRVAKILI S M, SIM D, HUNTER I W, et al. Actuation of untethered pneumatic artificial muscles and soft robots using magnetically induced liquid-to-gas phase transitions[J]. Science Robotics, 2020，5(41): eaaz4239.

[90] BERSELLI G, PICCININI M , VASSURA G. Comparative evaluation of the selective compliance in elastic joints for robotic structures[C]// 2011 IEEE International Conference on Robotics and Automation, Shanghai, China, IEEE, 2011: 4626-4631.

[91] GRUBER S. Robot hands and the mechanics of manipulation[J]. IEEE, 1987, 75(8): 1134-1134.

[92] LIAROKAPIS M, DOLLAR A M. Deriving dexterous, in-hand manipulation primitives for adaptive robot hands[C]// 2017 IEEE/RSJ International Conference on Intelligent Robots and Systems, Vancouver, BC, IEEE, 2017: 1951-1958.

[93] 张玉茹, 李继婷, 李剑锋. 机器人灵巧手: 建模, 规划 与仿真[M]. 北京: 机械工业出版社, 2007.

ZHANG Yuru, LI Jiting, LI Jianfeng. Robot dexterous hands: Modeling, planning and simulation[M]. Beijing: China Machine Press, 2007.

[94] DONG Huixu, ASADI E, QIU Chen, et al. Geometric design optimization of an under-actuated tendon-driven robotic gripper[J]. Robotics and Computer-Integrated Manufacturing, 2018, 50: 80-89.

[95] NUZZI C, PASINETTI S, LANCINI M, et al. Deep learning-based hand gesture recognition for collaborative robots[J]. IEEE Instrumentation and Measurement Magazine, 2019, 22(2): 44-51.

[96] CHAO Ya, CHEN Xingchen, XIAO Nanfeng. Deep learning-based grasp-detection method for a five-fingered industrial robot hand[J]. IET Computer Vision, 2019, 13(1): $61-70$

[97] ANDRYCHOWICZ M, BAKER B, CHOCIEJ M, et al. Learning dexterous in-hand manipulation[J]. The International Journal of Robotics Research，2020，39(1): 3-20.

[98] LI Zhixiong, ZHANG Ziyang, SHI Junchuan, et al. Prediction of surface roughness in extrusion-based additive manufacturing with machine learning $[\mathrm{J}]$. Robotics and Computer-Integrated Manufacturing, 2019, 57: 488-495.

[99] OZAWA R, TAHARA K. Grasp and dexterous manipulation of multi-fingered robotic hands: A review from a control view point[J]. Advanced Robotics, 2017, 31(19-20): 1030-1050.

[100] SMIT G, PLETTENBURG D H, VAN DER HELM F C $\mathrm{T}$, et al. The lightweight Delft Cylinder Hand: First multi-articulating hand that meets the basic user requirements[J]. IEEE Transactions on Neural Systems and Rehabilitation Engineering, 2015，23(3): 431-440.

[101] WU F Y, ASADA H H. "Hold-and-manipulate" with a single hand being assisted by wearable extra fingers[C]// 2015 IEEE International Conference on Robotics and Automation, Seattle, Washington, IEEE, 2015 : 6205-6212.

作者简介: 蔡世波, 男, 1981 年出生, 博士, 副研究员, 博士研究生导 师。主要研究方向为机器人多指灵巧手、康复机器人。

E-mail: ccc@zjut.edu.cn

陶志成, 男, 1995 年出生, 硕士研究生。主要研究方向为多指灵巧手设 计、抓持与操作理论。

E-mail: 1059145862@qq.com

万伟伟, 男, 1985 年出生, 博士, 副教授, 博士研究生导师。主要研究 方向为机器人操作理论与方法。

E-mail: wan@sys.es.osaka-u.ac.jp

喻豪勇, 男, 1966 年出生, 博士, 副教授, 博士研究生导师。主要研究 方向为医疗康复机器人、机器人臂手系统。

E-mail: bieyhy@nus.edu.sg

鲍官军(通信作者), 男, 1979 年出生, 博士, 教授, 博士研究生导师。 主要研究方向为机器人多指灵巧手、仿生操作理论、软体机器人。

E-mail: gjbao@zjut.edu.cn 